\title{
MEASUREMENT AND ADJUSTMENT OF THE DIFFERENTIAL POSITIONS OF COLLIDING BUNCHES AT CESR*
}

\author{
M. Billing ${ }^{\dagger}$, G. Codner, R. Meller, J. Sikora, Cornell University, LNS, Ithaca NY 14853, USA
}

\section{Abstract}

In CESR, 9 trains of 4 or 5 bunches of electrons and positrons collide at the interaction point, but bypass each other in the arcs of the storage ring using electrostatically separated orbits (pretzels). The parasitic bunch crossings resulting from the use of pretzel separation causes the bunches to have different orbits and, thus, the luminosity to be sensitive to the relative transverse positions of colliding bunches. We measure the bunch-by-bunch position projected to the interaction point from information available from the transverse feedback systems used for beam stabilisation. Some modification of the feedback systems has permitted the application of a bunch-by-bunch deflection designed to adjust the relative positions of colliding bunches. Observation of bunch-bybunch position during collisions and the effect of relative position adjustments will be presented.

\section{INTRODUCTION}

In accelerators which collide bunches with small vertical beam sizes, errors in the vertical differential positions of the two beams will result in the failure of the bunch distributions to overlap completely. This effect can cause a reduction in the luminosity due not only to the incomplete vertical overlap but also to the generation of an overall vertical deflection of one beam by the other creating beam-beam resonance's which would be otherwise missing by symmetry. Great care is generally taken to adjust the average positions of the beams relative to each other. However, the fact the accelerators usually have a large number of bunches and these bunches may have different orbits implies that it is likely that some colliding bunch differential (positron minus electron) positions will not be optimum.

In the single vacuum chamber for CESR there are typically 9 trains of 5 bunches circulating in each beam, having a vertical beam size of $4-5 \mu \mathrm{m}$ vertically. The bunches are separated from those in the counter-rotating beam using electrostatic horizontal and vertical separators. Observations of the optimum vertical position from beam-beam luminosity deflection measurements (best overlap of the colliding bunches) indicate that bunches within a train have systematically different orbits[1,3]. This can arise from the different beam-beam deflections occurring at the parasitic (separated) collision points in the ring.

To attempt to correct these bunch-by-bunch orbit

\footnotetext{
* Work supported by the National Science Foundation.

$\dagger$ mgb@cesr10.1ns.cornell.edu
}

differences a kicker capable of deflecting each bunch independently is needed. The transverse feedback kickers in CESR are used to stabilize the beam by independently deflecting each bunch an amount determined by the betatron oscillation displacement for that bunch and are thus a possible deflection element to reduce the bunch-bybunch orbit differences. By design, the feedback systems' processors suppress any deflection which is constant for each bunch. However, the software for the feedback systems can be easily reconfigured to allow a constant deflection to be programmed for each bunch. So if the feedback kicker location is approximately an odd number of quarter betatron wavelengths from the interaction point (IP), it will be possible to deflect the beam transversely on a bunch-by-bunch basis.

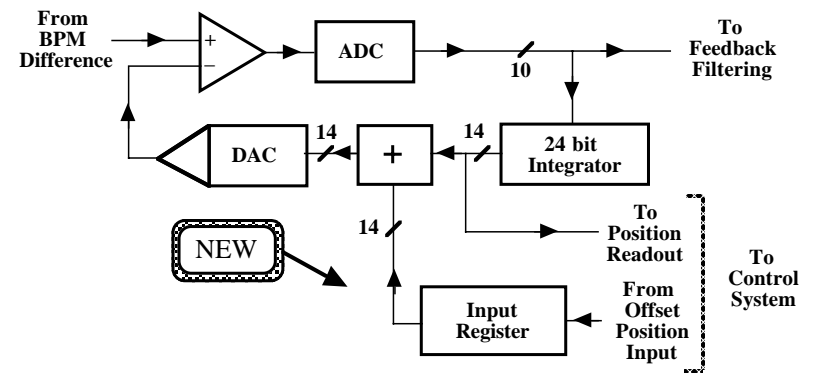

Figure 1. Block Diagram of feedback system modification.

\section{FEEDBACK SYSTEM MODIFICATIONS}

The CESR feedback system[2] uses a beam position monitor (BPM) 1.16 betatron wavelengths $\left(\lambda_{\beta}\right)$ from the IP as the sum (intensity) and difference (position) inputs to its sample and hold circuits and analog-to-digital converters (ADC's.) After digitisation, an integrated average for each input is calculated and stored memory. These averages are read out by the control system for monitoring the intensity and position times intensity for all bunches. Also the intensity value is available for use by feedback processor for current normalisation of the difference signal. The position average is subtracted from the position signal turn-by-turn to remove a constant orbit position and to give the betatron dipole displacement times intensity turn-by-turn. Since the feedback processors' filtering has a non-zero response at DC by design, this subtraction removes for each bunch any constant kick, which would needlessly use feedback amplifier power. If the BPM is a multiple of half $\lambda_{\beta}$ from the IP, the difference of the average position signal for each colliding bunch pair permits the calculation of the their separation at the IP. There are likely to be different offsets for each bunch in each beam e.g. due to crosstalk of the raw BPM signal and any beam-beam kicks at 
intervening parasitic crossings. Having the ability to monitor relative changes in the positions of colliding bunches is still useful as an on-line monitor when bunchby-bunch kicking is in operation. As is shown in figure 1 , the feedback processing has been modified to have a memory location, able to be written into by the accelerator control system. This value is subtracted from the average position, allowing a constant deflection to be applied independently by the feedback kicker to each bunch.

\section{OPTICS CONSIDERATIONS}

For the bunch-by-bunch kick from the feedback kicker to be effective at changing the separation of the colliding bunches at the IP, certain approximate optics relations are required. At CESR's operating vertical tune, the optimum condition for the kicker would be for it to be approximately an odd number of one-quarter $\lambda_{\beta}$ from the IP. As mentioned above the optimum condition for monitoring the separation of colliding bunches at the IP would have the BPM a multiple of half $\lambda_{\beta}$ from the IP. In both cases the larger the vertical beta functions, the more sensitive the kick and position measurement.

At the full feedback amplifier output, the $1.05 \mathrm{~m}$ stripline kicker develops $+/-200 \mathrm{~V}$ on its $0.1 \mathrm{~m}$ spaced plates. With the beam at $5.3 \mathrm{GeV}$ this represents a maximum deflection of $0.8 \mu \mathrm{rad}$. Using the typical design parameters for CESR found Table 1, this gives a maximum differential vertical displacement at the IP of $0.43 \mu \mathrm{m}$ for one pair of bunches. In operation it is the separation difference between pairs of bunches that is important. This is the case since there is already a mechanism for separating all bunches in the beam at the IP by the same amount using a differential orbit ripple from unclosing the vertical separation bump needed at the point in CESR diametrically opposite to the IP. This control is known as "VCROSING 7." So changing the separation of one pair of bunches maximally relative to another pair is done by making oppositely signed relative beam displacements for both pairs of bunches and then correcing the average separation using VCROSING 7.

Table 1: Typical feedback system optics design parameters for CESR

\begin{tabular}{|l|c|c|}
\hline & Beta Vert & $\begin{array}{c}\text { Vert. Phase } \\
\text { Advance from IP }\end{array}$ \\
\hline Kicker & $22.9 \mathrm{~m}$ & $1.68 \lambda_{\beta}$ \\
\hline BPM & $20.7 \mathrm{~m}$ & $1.16 \lambda_{\beta}$ \\
\hline IP & $0.018 \mathrm{~m}$ & $0 \lambda_{\beta}$ \\
\hline Vert. Tune & \multicolumn{2}{|c|}{10.58} \\
\hline
\end{tabular}

With a phase advance from the BPM to the IP of 1.16 $\lambda_{\beta}$, the position measurement made by the feedback system will be sensitive at the IP to differential vertical angles in addition to the differential vertical position. The
BPM's phase advance to the IP not being optimum together with the fact that there are several parasitic crossings between the IP and the BPM render this tool less ideal for determining the actual separation of bunches at the IP.

\section{PERFORMANCE}

\subsection{Single Beam Studies}

During machine studies (MS) periods a number of measurements were undertaken to study the performance of this instrumentation. Using VCROSING 7 to displace the beam a known amount, the electron and positron BPM position readouts were separately calibrated. The position readouts are accessible at a $60 \mathrm{~Hz}$ rate and it has been found that averaging over 100 readings projects a position to the IP with an uncertainty of approximately $0.1 \mu \mathrm{m}$ vertically at currents of 5 to $8 \mathrm{~mA}$ per bunch. During these observations it was clear that the average position values were drifting slowly over a several minute time period, suggesting that there is a systematic (perhaps intensity dependant) unidirectional trend in either the vertical beam position or the readout electronics.

After calibrating the feedback system position monitoring, single beam measurements of the bunch-bybunch position crosstalk were made. Initially 9 trains of 4 bunches were filled to $0.2 \mathrm{~mA}$ per bunch which is above the threshold of sensitivity for the input electronics of the feedback system. Then, in sequence, the fourth, then the third, etc. bunches in all trains were filled and the shift of the average position of all trailing bunches was measured. At $6 \mathrm{~mA}$ per bunch the feedback electronics projected an average vertical position shift at the IP for all trailing bunches of $+0.7+/-0.2 \mu \mathrm{m}$ for positrons and $+1.7+/-0.2$ $\mu \mathrm{m}$ for electrons as the bunch $14 \mathrm{nsec}$ immediately preceding was filled. This effect was not cumulative, i.e. the results are consistent with there being no shift in position when a bunch $28 \mathrm{nsec}$ or earlier was filled. Since the shift apparently only occurred when the bunch immediately ahead is filled, the suspicion is that it is likely that this is some form of BPM signal reflection or electronic crosstalk rather than wakefields affecting the orbits.

Finally there were MS measurements of the vertical orbit motion when the bunch-by-bunch deflections were turned on. This was performed with 9 trains of 4 bunches of either species. During this study the change in the vertical positions of all four bunches, averaged over all trains, were measured for the case when all bunch 1's were deflected in one direction and all bunch 4's were deflected in the opposite sense. (Bunch's 2's and 3's were left unchanged.) For a full scale vertical deflection projected to the IP for these bunches, the differential position motion for bunch 1 relative to bunch 4 was $0.4-0.5 \mu \mathrm{m}$ for both positrons and electrons, as expected. The results are consistent with no motion for bunches 2 and 3 . 


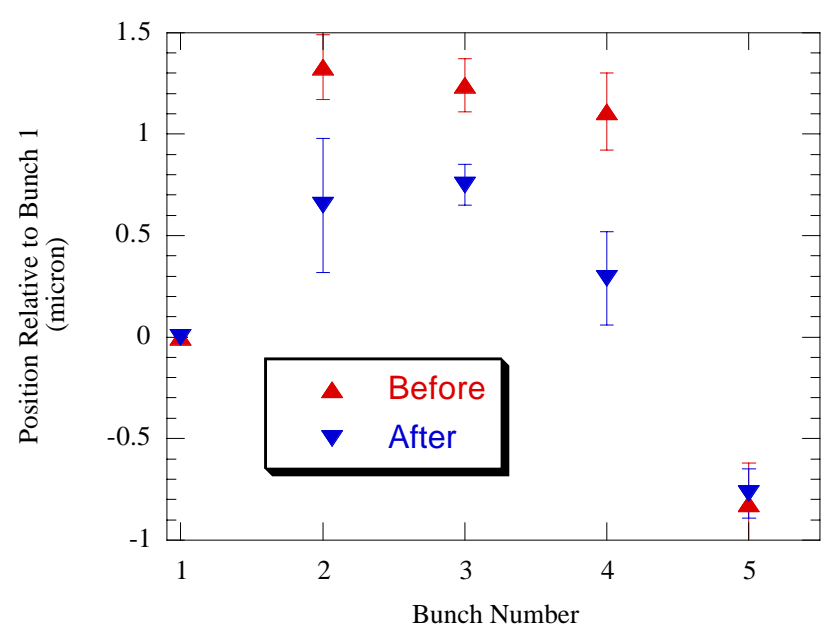

Figure 2. The positron-minus-electron vertical separation of the 5 bunches in the trains (averaged over all 9 trains)

when the first bunches have no separation, displayed before and after the bunch-by-bunch kicking was initiated.

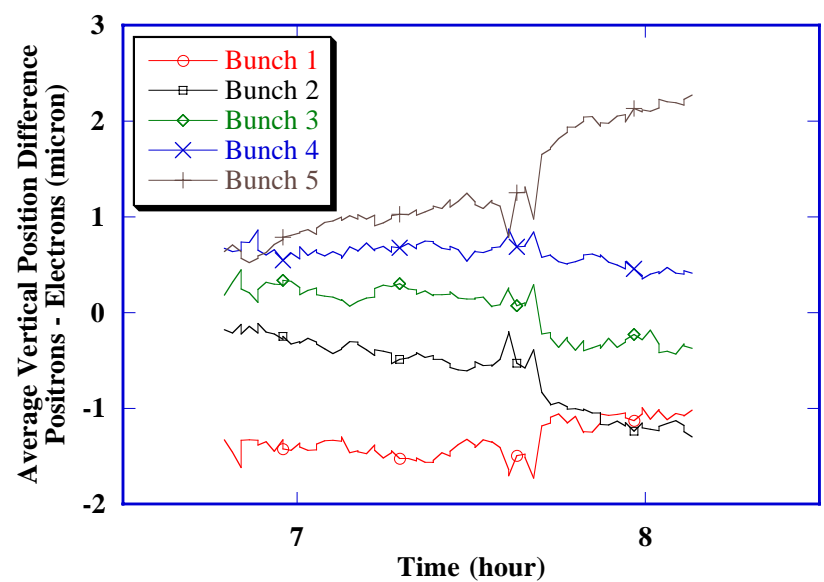

Figure 3. Plot of the positron-minus-electron vertical positions (averaged over all trains) measured at the feedback system BPM and projected as a displacement at the IP as recorded by the on-line logging system.

\subsection{Colliding Beam Studies}

With 9 trains of 4 bunches in two colliding beams during MS, bunches 1 and 4 of all trains of the positrons were again deflected in opposite directions to each other while bunches 1 and 4 of electrons were deflected in the opposite sense relative to the positron bunches. For a full scale kick, again the average separation projected to the IP was consistent with an $0.8 \mu \mathrm{m}$ change of the positron position relative to the electron position in one direction for all bunch 1's and in the opposite direction for all bunch 4's, as was expected.

During a few high energy physics (HEP) running periods, vertical bunch-by-bunch kicks were turned on in an attempt to reduce the average vertical separation of all colliding bunches. As described elsewhere[3], measurements of the vertical separation of colliding electron and positron bunches (as averaged over all 9 trains) were performed by scanning the VCROSING 7 control while observing the beam-beam kick monitor (BBKM.) The BBKM measures the strength of the beambeam interaction by displacing bunches of one beam and measuring the deflection coupled to the other beam. It has a peak response when the colliding bunches have a maximum vertical overlap[1]. The BBKM can be triggered to select one of the bunches in each train. The VCROSING 7 control used in tandem with the BBKM allows finding the vertical separation setting, which maximises the bunch-to-bunch vertical overlap for a certain bunch in all trains. This measurement results in the relative displacements of the centroids of the colliding bunches averaged over all trains.

Shown in figure 2 are the results of one set of HEP measurements with 9 trains of 5 bunches, where the relative vertical differences as measured using the VCROSING 7 control and the BBKM are shown before and after the bunch-by-bunch deflections were started. In this set of measurements in all trains bunches 1 and 5 had the positrons and electrons deflected toward each other and bunches 2 through 4 deflected away from each other. For all bunches the amplitude of the vertical deflections were at one half of the maximum so as to allow for additional vertical deflections from the BBKM system and the vertical feedback system. After the bunch-by-bunch kicks were installed, an overall vertical separation adjustment using VCROSING 7 brought bunches 2 through 4 back into optimum overlap. As is seen in figure 2 the relative positron-to-electron vertical displacements were changed by $0.6+/-0.3 \mu \mathrm{m}$. Figure 3 shows the positron minus electron positions measured by the feedback BPM and projected to the IP as a pure displacement. This data was recorded by the on-line logging software and shows the VCROSING measurements (just before 7:40 a.m.) and the bunch-by-bunch kicking being started (at 7:40 a.m.) This data also shows the offsets present in the measurements and the change in relative positions during an HEP fill.

\section{CONCLUSIONS}

A method of adjusting the vertical positron-electron separation bunch-by-bunch has been created and successfully studied using the vertical feedback system's kicker. In this implementation the maximum change of relative vertical separation produced by the kicker is about $20-25 \%$ of the beam's sigma and an increase of this displacement by 3 to 4 times would be desirable.

\section{REFERENCES}

[1] D. Sagan, J. Sikora, and S. Henderson, "A Luminosity Monitor via the Beam-Beam Interaction.", Cornell LNS Note: CBN 97-13.

[2] M. Billing, G. Codner, R. Meller, J. Sikora, "Performance of the Beam Stabilizing Feedback Systems at CESR", this conference.

[3] D. Sagan, M. Billing, M. Palmer, "Effect on Luminosity from Bunch-to-Bunch Orbit Displacements at CESR", this conference. 